\title{
An Analytical Model for IEEE 802.15.4/ ZigBee Wireless Sensor Networks with Duty Cycle Mechanism for Performance Prediction and Configuration of MAC Parameters to Achieve QoS and Energy Efficiency
}

\author{
Dushyanta Dutta ${ }^{1}$, Arindam Karmakar ${ }^{1}$ and Dilip Kr. Saikia ${ }^{2}$ \\ ${ }^{1}$ Department of Computer Science and Engineering, Tezpur University, India \\ ${ }^{2}$ Department of Computer Science and Engineering, National Institute of Technology Meghalaya, India
}

\begin{abstract}
The IEEE 802.15.4 standard defines a Physical (PHY) and Medium Access Control (MAC) layer protocol for low data rate wireless network with low power requirement which makes it ideal PHY/MAC for WSNs. Currently WSNs with combination of IEEE 802.15.4 and ZigBee are being used extensively in industrial applications such as factory automation and control, environmental monitoring etc. Such applications need Quality of Service (QoS) in terms of reliability and latency along with energy efficiency for longevity of the network.

The slotted IEEE 802.15.4 MAC protocol uses the duty cycle mechanism to save energy. However the duty cycle mechanism reduces the effective bandwidth leading to possibility of higher packet collision in the active periods and an improper choice of duty cycle may result in low packet delivery ratio and higher packet latency. Similarly there other MAC parameters such as the beacon interval which have bearing on performance of the WSN. It is therefore necessary to make accurate analysis of IEEE 802.15.4 MAC protocol, with duty cycle mechanism enabled, so that the MAC parameters such as Duty Cycle, beacon interval etc. can be set so as to ensure satisfaction of QoS requirements of applications while achieving maximum energy efficiency.

We present here an analytical model developed as a Markov Chain Process for slotted IEEE 802.15.4 MAC protocol assuming duty cycle mechanism is enabled in the protocol. Expressions are derived based on the model to compute percentage of packet successfully delivered within a given latency and the energy consumed by the sensor nodes in the process. The model is expected to be useful in deciding on the key MAC parameter values for applications of known traffic load and QoS requirements such as Packet Delivery Ratio(PDR) within given latency while maximizing energy efficiency. The analytical model presented is validated through simulation study in NS2.
\end{abstract}

\section{Keywords:}

Slotted IEEE802.15.4, Quality of Service (QoS), Packet Delivery Ratio (PDR), Energy Efficiency, Duty Cycle, Beacon Order

\section{INTRODUCTION}

The IEEE 802.15.4 standard has received considerable attention as a major low data rate and low power protocol for wireless sensor networks (WSNs) for applications like process control and factory automation which normally have very low rate traffic between sensors/actuators. Use of IEEE802.15.4 in WSNs is possible due to amalgamation of the IEEE 802.15.4 standard and the ZigBee specifications. The IEEE 802.15.4 standard [5] defines the physical and medium access control (MAC) layers and the ZigBee specification [11] defines the networking and application layers of the protocol stack.

For a successful deployment of WSNs in industrial environments along with energy efficiency, we have to deal with QoS parameters like successful delivery of data packets (reliability) and timeliness [12, 14]. In this paper a Markov Chain based analytical model is proposed to compute packet delivery ratio( $P D R)$ within a given latency and the energy consumed per byte of data delivered (EPBDD) in a WSN. The proposed model is validated with simulation results in NS2.

The rest of this paper is organized as follows. Section 2 discusses the related works. Section 3 introduces the IEEE 802.15.4 MAC protocol. Section 4 gives a detailed description of the Markov Chain Model proposed and equations derived based on the model to calculate PDR within a given latency limit and energy consumption of a node. In Section 5 we validate our proposed model by comparing analytical results with results obtain from NS2 simulation. Finally, Section 6 draws the conclusions of the work.

\section{RELATED WORK}

In recent years, some notable analysis have been presented based on results obtained from simulation on the performance of IEEE 802.15.4 WSNs when duty cycle mechanism is enabled.

The earliest work that studies the performance of the network in terms of throughput and overall power consumption of the network due to change in duty cycle is by Gang et al. [6]. A number of analytical models have also been developed considering the inactive period in the beacon interval for IEEE 802.15.4 MAC based on the Bianchi's model [1] developed for IEEE 802.11 DCF. These include both saturated and unsaturated conditions. Miic et al. [7] have developed an analytical model to study the throughput perfor- 
mance at different packet arrival rates, number of stations, buffer sizes, and packet sizes. Gao et al. [3], Xiao et al.|[13], and Huang et al.[4] also developed analytical model to study effect of inactive period on the performance of the network in terms of throughput and power consumption.

The existing works of analytical modeling and simulation focus mainly on the study of the impact of inactive period on the performance of IEEE 802.15.4 networks. However, no work has been observed that deals with QoS requirements of applications in terms of packet delivery ratio $(P D R)$ and packet latency together with energy efficiency when the duty cycle mechanism is enabled.

Park et al. [10] developed a model for IEEE 802.15.4; however the duty cycle mechanism was not considered in the model. Based on the model authors derive expressions to compute packet delivery ratio, average latency and power consumption. The work mainly focuses on the performance analysis and validating the accuracy of the proposed model.

A WSN application is characterized by its traffic load, required packet delivery ratio (PDR) and a latency limit. The two key MAC parameters that play important role in satisfying the requirements of the application are the duty cycle $(d c)$ and the beacon interval (bi). This work focuses on determining the combination of Duty Cycle and Beacon Interval for a WSN an application with given characteristics such that the consumed amount of energy per byte of data delivered $(E P B D D)$ is minimized.

Let the objective function $E_{P B}(d c, b i, p d r, \delta, l)$ denote average energy consumed per byte of data delivered with packet delivery ratio no less than $p d r$ and latency no more than $\delta$ at given traffic load $l$ for duty cycle $d c$ and beacon interval $b i$. The constrained optimization problem of the network is therefore-

$$
\text { minimize } E_{P B}(d c, b i, p d r, \delta, l)
$$

An analytical approach is used to solve the above optimization problem expressed in Eq.(1). The analytical model developed, computes packet delivery ratio $(P D R)$ achieved and the corresponding energy consumed by the WSN for the packets delivered within a given latency limit, considering that it as a vital for QoS satisfaction of applications. Further, for the energy consumed, we compute the energy per byte of data delivered (EPBDD) as it reflects the true energy efficiency.

\section{IEEE 802.15.4}

IEEE 802.15.4 PHY/MAC was originally designed for Personal Area Networks (PANs) [5]. An IEEE 802.15.4 network basically consists of a coordinator and associated nodes which are synchronized to the coordinator. The channel accesses of IEEE 802.15.4 MAC are done in two operational modes- beacon enabled mode and non-beacon enabled mode. In the beacon enabled mode IEEE 802.15.4 MAC operates as slotted Carrier Sense Multiple Access with Collision Avoidance (CSMA/CA) and in non-beacon enables mode it operates as unslotted CSMA/CA. The beacon enabled mode allows for an energy saving mechanism through use of the concept of Duty Cycle that provides for the transceivers in the nodes to be in Active or Sleep Modes in a synchronized manner. Here the transceivers are active during a Superframe that is bounded by a Beacon frame generated by the coordinator at regular interval. The interval between two consecutive beacons is the size of the beacon frame which is also called Beacon Interval $(\mathrm{bi})$. The duration of beacon interval is decided by the parameter called Beacon $\operatorname{Order}(B O)$ and $b i=15.36 * 2^{B O}$ ms where $0 \leq B O \leq 14$. The size of the active period is also called Superframe Duration $(S D)$ and its length is decided by the parameter Superframe Order

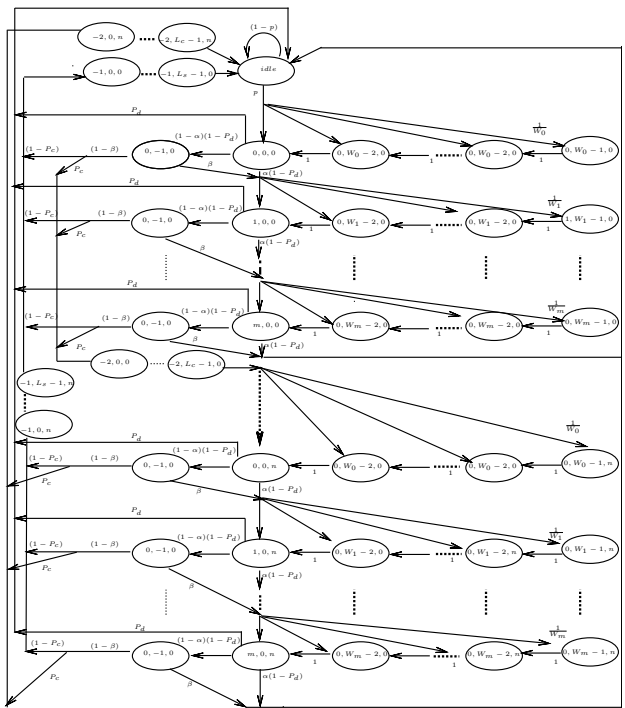

Fig. 1. Markov chain model for IEEE 802.15.4 and duty cycle mechanism with slotted CSMA/CA

Table 1. Summary of the notations used in the Analytical Model and Expressions

\begin{tabular}{|c|c|}
\hline$b_{i, k, j}$ & The probability of being at state $(i, k, j)$ in the Markov Chain \\
\hline$\lambda$ & Packet generation rate with Poisson distribution. \\
\hline$q_{0}$ & Probability of nodes obtaining a packet for transmission. \\
\hline$P P_{d}$ & $\begin{array}{l}\text { Probability that a packet is being differed due to lack of active period } \\
\text { in the current Beacon Inteval. }\end{array}$ \\
\hline$L$ & Total length of a packet in terms of backoff slots including overhead and payload. \\
\hline$t_{a c k}$ & Time a node will wait for the acknowledgement frame after transmission. \\
\hline & The length of an acknowledgement frame. \\
\hline$I F S$ & Inter-Frame Spacing. \\
\hline$t_{m, a c k}$ & Timeout of an acknowledgement frame. \\
\hline$W_{0}$ & Minimum backoff window size. \\
\hline$m_{0}$ & Minimum backoff exponent macMin $B E$. \\
\hline$m_{b}$ & Maximum backoff exponent $\operatorname{macMaxBE}$ \\
\hline$m$ & Maximum allowed backoff $\operatorname{macMaxCSMABackoffs.}$ \\
\hline$n$ & Maximum allowed retransmission macMaxFrameRetries. \\
\hline$\tau$ & Probability that a node senses the channel at CCA1 in a randomly chosen time slot. \\
\hline$p$ & Probability that a packet is ready for transmission. \\
\hline$P_{c}$ & Probability of a packet collision. \\
\hline$\alpha$ & Probability that a node finds the channel busy at CCA1. \\
\hline$\beta$ & Probability that a node finds the channel busy at CCA2. \\
\hline$N$ & Total number of contending nodes. \\
\hline$L_{S}$ & $\begin{array}{l}\text { Time required for successful transmission of a packet in terms number of backoff slots } \\
\text { where } L_{S}=L+t_{a c k}+L_{a c k}+I F S\end{array}$ \\
\hline$L_{C}$ & $\begin{array}{l}\text { Time spent for a collided packet in terms of number of backoff slots where } \\
L_{c}=L+t_{m}, a c k\end{array}$ \\
\hline $\bar{P}$ Pf & Probability that a packet is discarded due to channel access failure. \\
\hline$P_{c r}$ & Probability that a packet is discarded due to retry limits. \\
\hline$P_{i}$ & Power expenditures corresponding to the radios idle state. \\
\hline$P_{S C}$ & Power expenditures corresponding to the radios sensing state. \\
\hline$P_{t}$ & Power expenditures corresponding to the radios transmission state. \\
\hline$\overline{P r}$ & Power expenditures corresponding to the radios receive state. \\
\hline$P s p$ & Power expenditures corresponding to the radios sleep state. \\
\hline$P_{s i}$ & Power expenditures corresponding to the radios sleep to idle transition. \\
\hline$P_{i r}$ & Power expenditures corresponding to the radios idle to receive transition. \\
\hline$P_{i t}$ & Power expenditures corresponding to the radios idle to transmission transition. \\
\hline$T_{s i}$ & The time required by radio for sleep to idle transition. \\
\hline$T_{i r}$ & The time required by radio for idle to receive transition. \\
\hline$T_{i t}$ & The time required by radio for idle to transmission transition. \\
\hline$L_{B}$ & Duration of the beacon frame in terms of backoff slots which is 2 backoff slots. \\
\hline$f_{b}$ & The frequency of beacon reception which is computed as $f_{b}=\frac{1}{B I}$ \\
\hline$T_{b}$ & Probability that a node will be in state of receiving beacons. \\
\hline
\end{tabular}

( $S O$ ) and $S D=15.36 * 2^{S O} \mathrm{~ms}$, where $0 \leq S O \leq 14$. Thus the Duty Cycle $(D C)$ of a network operating in beacon enabled slotted CSMA/CA mode is $2^{(S O-B O)}$.

\section{THE MARKOV CHAIN MODEL}

The following are the changes in our model with reference to that in [10]: 
(1) Any transmission should be completed within the active period in a beacon interval, that is, if nodes realize that a transmission cannot be completed within the active period in the current beacon interval, it is postponed to the next beacon interval. We introduce the probability of a packet being deferred $P_{d}$ in the analytical model which is computed as

$$
P_{d}=\frac{L_{S}+2}{S D}
$$

(2) The term $q 0$ used in the model is defined as the probability that a node generates a packet during a slot. Packets are assumed to be of fixed $L$-backoff slot duration and arrive at the nodes according to a Poisson arrival rate of $\lambda$ packets per packet duration. Thus we compute this probability as

$$
q 0=\frac{\lambda}{L}
$$

(3) The analysis focuses on very low traffic so the effect of queue length is assumed here as negligible. Therefore in the proposed model the queue length in a node is not restricted unlike in [10] where a finite queue length is considered.

(4) We introduce the probability $p$ which is the probability that the node has packet for transmission. The packet ready for the transmission may be a newly generated packet or a packet that was deferred due to inactive period in the previous beacon interval. We compute this probability as-

$$
p=q 0+P_{d}
$$

(5) $Q_{0} \ldots Q_{L 0-1}$ used in [10] is replace with a single $i d l e$ state which represents the starting of a packet transmission process.

(6) As we consider starting of packet transmission process always start from idle state, node always goes to the idle state with probability 1 , after successful transmission or collision at maximum retransmission. Due to this consideration transition probability to idle state from the maximum backoff stage is either $\alpha\left(1-P_{d}\right)$ or $\beta$, on event of busy channel sensing.

\subsection{The Proposed Model}

The analytical model is a three dimensional Markov chain process which represents a node packet transmission process as indicated in Figure.1. It uses 3-tuples $<s(t), c(t), r(t)>$ to form the Markov chain process. Tuple $s(t), c(t)$ and $r(t)$ are representing the backoff stage, the state of the backoff counter and the state of retransmission counter at time $t$, respectively. States $\left(i, W_{m}-1, j\right)$ to $\left(i, W_{0}-1, j\right)$ are used to represent backoff stages and state $(i, 0, j),(i,-1, j)$ for CCA1, CCA2 and state $(-1, k, j)$, $(-2, k, j)$ for successful and unsuccessful packet transmission, respectively.

Proposition 1: Let the stationary distribution of the Markov chain in Fig 1 be $b_{i, k},{ }_{j}=\lim _{t \rightarrow \infty} \operatorname{Pr}(s(t)=i, c(t)=k, r(t)=j), i \in$ $(-2, m), k \in\left(-1, \max \left(W_{i}-1, L_{s}-1, L_{c}-1\right)\right), j \in(0, n)$. Then for $0 \leq i \leq m$

$$
b_{i, k},{ }_{j}=\frac{W_{i}-k}{W_{i}} b_{i}, 0,{ }_{j}
$$

where

$$
W_{i}=\left\{\begin{array}{cc}
2^{i} W_{0} & i \leq m_{b}-m_{0} \\
2^{m_{b}-m_{0}} W_{0} & i>m_{b}-m_{0}
\end{array}\right.
$$

and

$$
\begin{aligned}
& b_{0}, 0,0=\left[\frac{1}{2}\left(\frac{1-(2 x)^{m+1}}{1-2 x} W_{0}+\frac{1-x^{m+1}}{1-x}\right) \frac{1-y^{n+1}}{1-y}\right. \\
& \quad+\left(1-P_{d}\right)(1-\alpha)\left(\frac{1-x^{m+1}}{1-x}\right) \frac{1-y^{n+1}}{1-y}+\left(L_{s}\left(1-P_{c}\right)\right. \\
& \left.+L_{s} P_{c}\right)\left(1-P_{d}\right)(1-\alpha)(1-\beta)\left(\frac{1-x^{m+1}}{1-x}\right) \frac{1-y^{n+1}}{1-y} \\
& \quad+\frac{1}{p}\left[x^{m+1} \frac{1-y^{n+1}}{1-y}+\left(1-P_{d}\right)(1-\alpha)(1-\beta) P_{c}\left(\frac{1-x^{m+1}}{1-x}\right) y^{n}\right. \\
& +\left(1-P_{d}\right)(1-\alpha)(1-\beta)\left(1-P_{c}\right)\left(\frac{1-x^{m+1}}{1-x}\right) \frac{1-y^{n+1}}{1-y} \\
& \left.\left.\quad+P_{d}\left(\frac{1-x^{m+1}}{1-x}\right) \frac{1-y^{n+1}}{1-y}\right]\right]^{-1}
\end{aligned}
$$

Proof: First, we derive the state transition probability of Markov chain to compute the stationary probability. Secondly we apply the normalization condition to compute the probability $b_{0,0,0}$.

The state transition probabilities associated with the Markov chain are listed below-

$$
P(i, k, j \mid i, k+1, j)=1, \text { for } k \geq 0
$$

Eq.(7) is the decrement of backoff counter, which happens with probability 1 .

$$
P(i, k, j \mid i-1,0, j)=\frac{\left(1-P_{d}\right)(\alpha+(1-\alpha) \beta)}{W_{i}}, \text { for } i \leq m
$$

Eq.(8) represents the probability of finding busy channel either in CCA1 or CCA2 and of selecting a state uniformly in the next backoff stage provided there are available slots in active period for transmission.

$$
P(0, k, j \mid i, 0, j-1)=\frac{\left(1-P_{d}\right)(1-\beta)(1-\alpha) P_{c}}{W_{0}}, \text { for } j \leq n
$$

Eq.9 gives the unsuccessful transmission probability after finding an idle channel in both $C C A 1$ and $C C A 2$, and a node picks uniformly a state in the next retransmission stage.

$$
P(i d l e \mid m, 0, j)=\left(1-P_{d}\right)(\alpha+(1-\alpha) \beta) \text {, for } j<n
$$

Eqs. 10 represent the probability of going back to the idle stage due to the channel access failure.

$$
P(\text { idle } \mid i, 0, n)=\left(1-P_{d}\right)(1-\beta)(1-\alpha), \text { for } i<m
$$

Eqs.111 represent the probability of going back to the idle stage due to retry limits.

$$
P(i d l e \mid i, 0, j)=P_{d} \text {, for } i \leq m \text { and for } j \leq n
$$

Eq. 12 is the probability of going to the idle stage due to unavailable time slots in the active period for transmission at backoff counter $m$ and retransmission stage $n$.

$$
P(0, k, 0 \mid \text { idle })=\frac{p}{W_{0}}, \text { for } k \leq W_{0}-1
$$

Eq.13 models the probability of going back to the first backoff stage from the idle stage.

$$
P(\text { idle } \mid \text { idle })=(1-p)
$$


Eq. 14. models the probability that a node remains in idle stage. Based on Eqs. (7) to (14) we derive the closed form expression for $b_{i, k},{ }_{j}$ which is expressed in Eq. 5 .

From Eq. [8], for $i \leq m$ we obtain

$$
b_{i, 0,{ }_{j}}=\left(\left(1-P_{d}\right)(\alpha+\beta(1-\alpha))^{i} b_{0,0},_{j}\right.
$$

From Eq9 we can rewrite $b_{i, 0, j}$ as

$$
\begin{aligned}
& b_{0,0},{ }_{j}=\left(1-P_{d}\right)(1-\alpha)(1-\beta) P_{c} \sum_{i=0}^{m} b_{i}, 0,,_{j-1} \\
= & \left(\left(1-P_{d}\right)(1-\alpha)(1-\beta) P_{c} \sum_{i=0}^{m}\left(\left(1-P_{d}\right)(\alpha-\beta(1-\alpha))^{i}\right)^{j} b_{0}, 0,0\right.
\end{aligned}
$$

Next, we apply the normalization condition to derive expression for computing $b_{0,0,0}$. By the normalization condition, we know that

$$
\begin{aligned}
& \sum_{i=0}^{m} \sum_{k=0}^{W_{i}-1} \sum_{j=0}^{n} b_{i},_{k},_{j}+\sum_{i=0}^{m} \sum_{j=0}^{n} b_{-1,0},_{j} \\
& +\sum_{j=0}^{n}\left(\sum_{k=0}^{L_{s}-1} b_{-1,,_{k}, j}+\sum_{k=0}^{L_{c}-1} b_{-2, k_{k}, j}\right)+i d l e=1
\end{aligned}
$$

In Eq.(17), first term represents that the node is in backoff, second in sensing, third in packet transmission and fourth in idle state respectively.

We derive expressions to compute these terms, starting by the first term. Based on Eqs.(5), (16) and (17) we derive the expression to compute the probability, that the node is in backoff state which is outlined below-

$$
\begin{aligned}
& \sum_{i=0}^{m} \sum_{k=0}^{W_{i}-1} \sum_{j=0}^{n} b_{i},,_{j} \\
& =\sum_{i=0}^{m} \sum_{j=0}^{n} \frac{W_{i}+1}{2}\left(\left(1-P_{d}\right)(\alpha+\beta(1-\alpha))^{i} b_{0,0},{ }_{j}\right. \\
& =\left\{\begin{array}{l}
\text { if } m \leq m_{b}-m_{0} \\
\frac{b_{0}, 0,0}{2}\left(\frac{1-(2 x)^{m+1}}{1-2 x} W_{0}+\frac{1-x^{m+1}}{1-x}\right) \frac{1-y^{n+1}}{1-y} \\
\text { if } m>m_{b}-m_{0} \\
\frac{b_{0}, 0,0}{2}\left(\frac{1-(2 x)^{m_{b}-m_{0}+1}}{1-2 x} W_{0}+\frac{1-x^{m_{b}-m_{0}+1}}{1-x}\right. \\
\left.+\left(2^{m_{b}}+1\right) x^{m_{b}-m_{0}+1}\left(\frac{1-x^{m-m_{b}+m_{0}}}{1-x}\right)\right) \frac{1-y^{n+1}}{1-y}
\end{array}\right.
\end{aligned}
$$

where

$x=\left(1-P_{d}\right)(\alpha+\beta(1-\alpha))$

$y=\left(1-P_{d}\right)(1-\alpha)(1-\beta) P_{c}\left(\frac{1-x^{m+1}}{1-x}\right)$
The expression to compute the probability that the node is in channel sensing state is derived as:

$$
\begin{gathered}
\sum_{i=0}^{m} \sum_{j=0}^{n} b_{i},,_{-1},{ }_{j} \\
=\sum_{i=0}^{m} \sum_{j=0}^{n}(1-d)(1-\alpha)\left(\left(1-P_{d}\right)(\alpha+\beta(1-\alpha))\right)^{i} b_{0}, 0,{ }_{j} \\
=\left[\left(1-P_{d}\right)(1-\alpha)\left(\frac{1-x^{m+1}}{1-x}\right) \frac{1-y^{n+1}}{1-y}\right] b_{0}, 0,0
\end{gathered}
$$

The expression to compute the probability that the node is in transmission state is derived as:

$$
\begin{gathered}
\sum_{j=0}^{n}\left(\sum_{k=0}^{L_{s}-1} b_{-1, k},_{j}+\sum_{k=0}^{L_{c}-1} b_{-2},,_{j}\right) \\
=\left[\left(L_{s}\left(1-P_{c}\right)+L_{s} P_{c}\right)\left(1-P_{d}\right)(1-\alpha)(1-\beta)\left(\frac{1-x^{m+1}}{1-x}\right)\right. \\
\left.\times\left(\frac{1-y^{n+1}}{1-y}\right)\right] b_{0}, 0,0
\end{gathered}
$$

Lastly, we derive the expression to compute the probability that the node is in idle state. This is derived by considering that the successful transmission and the failure events are due to the limited number of backoff stages $m$ and the retry limits $n$ which is shown below:

$$
\begin{aligned}
\text { idle } & =\frac{1}{p}\left[x^{m+1}\left(\frac{1-y^{n+1}}{1-y}\right)+\left(1-P_{d}\right)(1-\alpha)(1-\beta) P_{c}\left(\frac{1-x^{m+1}}{1-x}\right) y^{n}\right. \\
& +\left(1-P_{d}\right)(1-\alpha)(1-\beta)\left(1-P_{c}\right)\left(\frac{1-x^{m+1}}{1-x}\right) \frac{1-y^{n+1}}{1-y} \\
& \left.+P_{d}\left(\frac{1-x^{m+1}}{1-x}\right) \frac{1-y^{n+1}}{1-y}\right] b_{0}, 0,0
\end{aligned}
$$

By replacing Eqs. (18)-21] in Eq. (17), we obtain the expression for $b_{0,0,0}$ which is shown in Eq. 6.

We derive the probability of successful packet reception, or reliability [10] which is expressed in Eq. 22]. This expression will be used later on while computing successful packet reception, or reliability within a given latency limit.

$$
P D R=1-P_{c f}-P_{c r}
$$

In order to derive the probability of successful reception we need the expressions of carrier sensing probability $\tau$, collision probability $P_{c}$ and busy channel probabilities $\alpha$ and $\beta$. Expressions for computing these probabilities remain same as [10]. These expressions, all together form a system of non-linear equations which is solved numerically using the Fixed Point Iteration method.

\subsection{Packet Delivery Ratio within a given Latency and the Energy Consumed by a node:}

We derive here the expressions to compute Packet Delivery Ratio (PDR) achieved within a given latency and the Energy Consumed by a node. To derive the expression for computing PDR 
achieved within a given latency we need the delay distribution of successfully received packet. The distribution of delay is obtained by expressing the Probability Generating Function (PGF) of delay experienced by a successfully received packet [7], [9]. To start with, the PGF for the effective duration of the backoff period, considering the presence of inactive period in the time frame.

Proposition 2: Let $B_{o f f}(Z)$ be the PGF for the effective duration of the backoff period, considering the presence of inactive period then we can compute $B_{o f f}(Z)$ as

$$
B_{\text {off }}(Z)=\left(1-P_{\text {last }}\right) Z+P_{\text {last }} Z^{(B I-S D)} B_{e}(Z)
$$

Proof: Eq. 23 consists of two terms which are the PGF's, representing that backoff countdown process during active and inactive period. The backoff counter decrements by one slot at the active period and freezed during inactive period. The PGF for active and inactive period are computed as $\left(1-P_{\text {last }}\right) Z$ and $P_{\text {last }} Z^{(B I-S D)} B_{e}(Z)$, respectively. The term $P_{\text {last }}$, is the probability that backoff period is the last within the superframe and it is expressed as-

$$
P_{\text {last }}=\frac{1}{S D}
$$

A beacon frame is received before resuming the backoff countdown, so term $P_{\text {last }} Z^{(B I-S D)}$ is product with $B_{e}(Z)$. The term $B_{e}(Z)$, is the PGF for the duration of the beacon frame which is derived as-

$$
B_{e}(Z)=Z^{2}
$$

Since, during the backoff process, node finds its radio either in active or sleep state, therefore $B_{o f f}(Z)$ is obtain as summation of terms $P_{\text {last }} Z^{(B I-S D)}$ and $P_{\text {last }} Z^{(B I-S D)} B_{e}(Z)$.

Proposition 3: Let $T_{\text {succ }}(Z)$ be the PGF for successful transmission considering the presence of inactive period and $T_{c o l}(Z)$ for unsuccessful transmission respectively. They are computed as:

$$
\begin{aligned}
& T_{\text {succ }}(Z)=\left(1-P_{d}\right) Z^{L_{s}}+P_{d} B_{p}(Z) Z^{(B I-S D)} B_{e}(Z) Z^{L_{s}} \\
& T_{c o l}(Z)=\left(1-P_{d}\right) Z^{L_{c}}+P_{d} B_{p}(Z) Z^{(B I-S D)} B_{e}(Z) Z^{L_{c}}
\end{aligned}
$$

Proof: Eq. 26, consist of two terms which are the PGF's for non deferred and deferred successful transmission which are computed as $\left(1-P_{d}\right) Z^{L_{s}}$ and $P_{d} B_{p}(Z) Z^{(B I-S D)} B_{e}(Z) Z^{L_{s}}$ respectively. The term $Z^{L_{s}}$ is the PGF for successful packet transmission time. The term $B_{p}(Z)$ is the PGF for $L_{s}+1$ slots which are wasted due to the insufficient slots in the current superframe and it is computed as:

$$
B_{p}(Z)=\frac{1}{L_{s}+1} \sum_{k=0}^{L_{s}} Z^{k}
$$

A node waits for $(B I-S D)$ slots during inactive period and its PGF is computed as $Z^{(B I-S D)}$. Before resuming the transmission process node receives a beacon frame, so the term $Z^{(B I-S D)}$ is product with $B_{e}(Z)$. Since a packet transmitted successfully is either a deferred or non deferred packet, therefore $T_{\text {succ }}(Z)$ is obtain as summation of terms $\left(1-P_{d}\right) Z^{L_{s}}$ and $P_{d} B_{p}(Z) Z^{(B I-S D)} B_{e}(Z) Z^{L_{s}}$.

The above mentioned mechanism also holds for an unsuccessful packet and its PGF is shown in Eq. 27). The term $Z^{L_{c}}$ used in
Eq. 27 is the PGF for unsuccessful packet transmission time.

Proposition 4: Let $D(Z)$ be the PGF of delay experienced by a packet to be successfully received. The PGF of the packet delay considering the effects of inactive period, backoff period, transmission and retransmission with maximum allowed backoff of $m$ and retransmission $n$ can be express as:

$$
\begin{aligned}
& D(Z)=T_{\text {succ }}(Z) \sum_{j=0}^{n}\left(1-P_{c}\right) P_{c}^{j} T_{\text {col }}(Z)^{j} \\
& \left(\sum_{i=0}^{m}\{1-(1-\alpha) \beta\}\{(1-\alpha) \beta\}^{i} S_{c}(Z)^{i+1} H_{i+1}(Z)\right)^{j+1}
\end{aligned}
$$

Proof: $D(Z)$ is derived by considering the Markov chain shown in Fig 1 Transmission occurs when the channel is being sensed idle for two CCAs, and its PGF is represented by term $S_{c}(Z)=Z^{2}$ as it requires two slots for CCA1 and CCA2. We consider node always finds the channel idle at CCA1 and goes for channel sensing in CCA2. A packet transmitted is successful with probability $\left(1-P_{c}\right)$. On event of collision node initializes the retransmission procedure with probability $P_{c}$. The retransmission procedure continues for maximum retransmission limit of $n$.

Since packet is transmitted after channel is sensed idle during two CCAs, the probability for channel idleness is $\{1-(1-\alpha) \beta\}$. It initializes the next backoff procedure when it finds the channel busy during CCA2 with probability $(1-\alpha) \beta$. The backoff mechanism continues for maximum backoff limit of $m$.

Consider the node to be at $i^{t h}$ backoff stage. At the end of backoff it senses the channel busy with probability $\{(1-\alpha) \beta\}^{i}$ and goes for the next backoff stage. The PGF of the random backoff process at $i^{\text {th }}$ backoff stage is represented by the term $H_{i}(Z)$ and is expressed as the product from $0^{t h}$ to $i^{\text {th }}$ stage:

$$
H_{i}(Z)=\prod_{k=0}^{i} W_{k}(Z)
$$

where $W_{k}(Z)$ is the PGF of the backoff time at the $i^{t h}$ backoff stage which is:

$$
W_{i}(Z)=\left\{\sum_{k=0}^{2^{i} W_{0}-1} \frac{1}{2^{i} W_{0}} B_{o f f}^{k}(Z)=\frac{B_{o f f}^{2^{i} W_{0}}(Z)-1}{2^{i} W_{0}\left(B_{o f f}(Z)-1\right)}\right.
$$

The node senses the channel idle with probability $\{1-(1-\alpha) \beta\}\{(1-\alpha) \beta\}^{i}$ at $(i+1)^{t h}$ backoff stage and transmits the packet after waiting $\sum_{i=0}^{m} S_{c}(Z)^{i+1} H_{i+1}(Z)$ due to $i$ number of busy channel sensing. Consider the packet transmitted as the $j^{\text {th }}$ transmission. On event of collision with probability $P_{c}^{j}$ at $j^{\text {th }}$ transmission attempt, it initiates the next retransmission procedure. The successful transmission occurs with probability $\left(1-P_{c}\right) P_{c}^{j}$ at $(j+1)^{t h}$ attempt after waiting $\sum_{j=0}^{n} T_{\text {col }}(Z)^{j}\left(\sum_{i=0}^{m}\{1-(1-\alpha) \beta\}\{(1-\alpha) \beta\}^{i} S_{c}(Z)^{i+1} H_{i+1}(Z)\right)^{j+1}$, for $j$ number of collision and will spend $T_{\text {succ }}(Z)$ as successful packet transmission.

Proposition 5: Let $D_{L_{M}}(1)$ be the PGF for successfully received packet with delay within limit $L_{M}$. The expression to compute expected Packet Delivery Ratio (PDR) within a given delay limit $L_{M}$ 
can be expressed as

$$
P D R \times D_{L_{M}}(1)
$$

Proof: We can represent $D(Z)$ as a power series where $T_{d}$ is the delay experienced by a successfully received packet.

$$
D(Z)=\sum_{i=0}^{\infty} \operatorname{Pr}\left(T_{d}=i\right) Z^{i}
$$

We can rewrite the Eq. 32 as $D_{L_{M}}(Z)=\sum_{i=0}^{L_{M}} \operatorname{Pr}\left(T_{d}=i\right) Z^{i}$. From this function we can find the PDR within a given latency limit $L_{M}$. Approximately it is the product of PDR and $D_{L_{M}}(1)$.

Proposition 6: The average Energy Consumption of a sensor node in the network is:

$$
\begin{aligned}
& P_{s p}\left(i d l e+\frac{B I-S D}{B I}\right)+P_{r} T_{b}+P_{s i} T_{s i}\left(f_{b}+p\right) \\
& +P_{i r} T_{i r} \sum_{i=0}^{m} \sum_{j=0}^{n} b_{i,,_{0},{ }_{j}}+P_{i t} T_{i t} \sum_{j=0}^{n}\left(b_{-1,0},_{j}+b_{-2,0},,_{j}\right) \\
& +P_{i} \sum_{i=0}^{m} \sum_{k=0}^{W_{i}-1} \sum_{j=0}^{n} b_{i,{ }_{k},{ }_{j}}+P_{s c} \sum_{i=0}^{m} \sum_{j=0}^{n}\left(b_{i},,_{j}+b_{i,,_{-1},{ }_{j}}\right) \\
& +P_{t} \sum_{j=0}^{n} \sum_{k=0}^{L-1}\left(b_{-1, k},{ }_{j}+b_{-2,{ }_{k},{ }_{j}}\right)+P_{i} \sum_{j=0}^{n}\left(b_{-1,{ }_{L},{ }_{j}}+b_{\left.-2,{ }_{L},{ }_{j}\right)}\right) \\
& +\sum_{j=0}^{n} \sum_{k=L+1}^{L+L_{a c k}+1}\left(P_{r} b_{-1, k},{ }_{j}+P_{i} b_{-2, k}, j\right)
\end{aligned}
$$

Proof: The expression to compute the average Energy Consumption by a node is the amount of energy consumed at different states. The first term in Eq. 33, consider energy consumption during idle and sleep state. The second term considers the energy consumption during beacon reception. Third, fourth and fifth terms consider energy consumption during transition of radio from sleep to idle, idle to receive and idle to transmit state, respectively. The sixth and seventh terms consider energy consumption during backoff and channel sensing state, respectively. The eighth, ninth and tenth terms consider the energy consumption during packet transmission stage. The expression for computing term, sixth, seventh, eighth, ninth and tenth is kept same as [10]. The probability that a node will be in state of receiving beacon frame is computed as $T_{b}=L_{B} f_{b}$. The probability that a node will be in state of transition from sleep to idle, idle to receive and idle to transmission are computed as $T_{s i}\left(f_{b}+p\right), T_{i r} \sum_{i=0}^{m} \sum_{j=0}^{n} b_{i, 0},{ }_{j}$ and $T_{i t} \sum_{j=0}^{n}\left(b_{-1,{ }_{0},{ }_{j}}+b_{\left.-2,,_{0},{ }_{j}\right) \text { re- }}\right.$ spectively.

\section{MODEL VALIDATION}

To validate the model simulated experiments have been carried out on NS-2 [8]. In all the experiments it is assumed that the IEEE 802.15.4 MAC protocol operating on top of the $2.4 \mathrm{GHz}$ physical layer with a maximum capacity of $250 \mathrm{Kbps}$. We consider a single hop star topology where all the stations are in radio range of each other and the coordinator is acting as the sink node. The network is operated in the beacon enabled mode and the retransmission mechanism is enabled. As the duty cycle mechanism is relevant to low traffic rates the data generation rate considered ranged between $0.25 \mathrm{Kbps}$ to $2.5 \mathrm{Kbps}$ which correspond to $0.1 \%$ and $1 \%$ of the maximum capacity. The data generation is considered to be Poisson distributed. The size of the packet is considered to be a fixed size of 100 bytes. The simulation is conducted in simulation time frame of 1000 s and results considered are aggregate value obtained over 100 runs of the simulation. For computation of the energy consumed by a sensor node we adopt the power consumption model of Chipcon CC2420 radio transceiver [2].

\subsection{Validation of the expression to compute PDR within a given delay limit:}
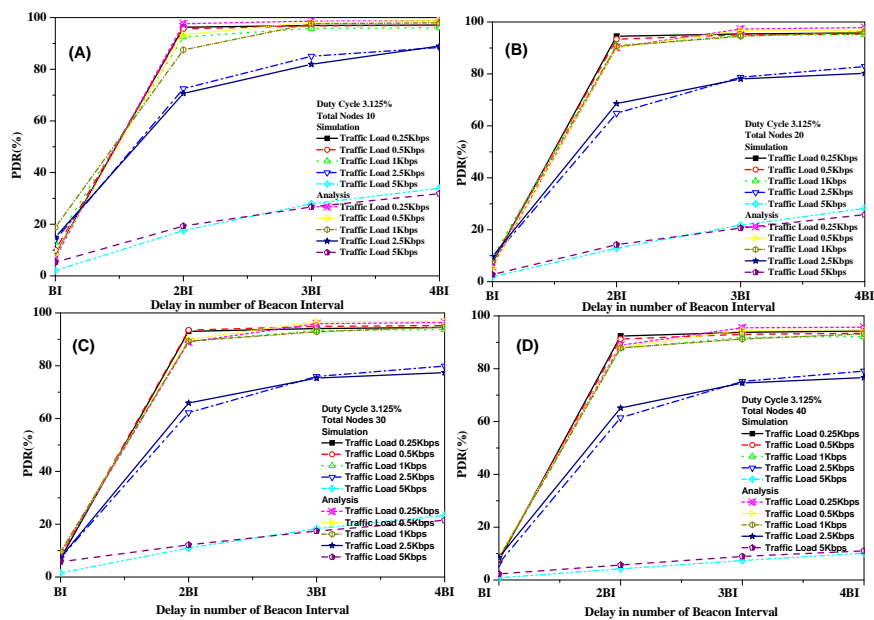

Fig. 2. PDR (\%) against burst number for both Simulation Results and Analytical Model at traffic load $0.25 \mathrm{Kbps}, 0.5 \mathrm{Kbps}, 1 \mathrm{Kbp}, 2.5 \mathrm{Kbps}$ and $5 \mathrm{Kbps}$ for Duty Cycle $3.125 \%$ as a function of total participating nodes (A) 10, (B) 20, (C) 30 and (D) 40 .
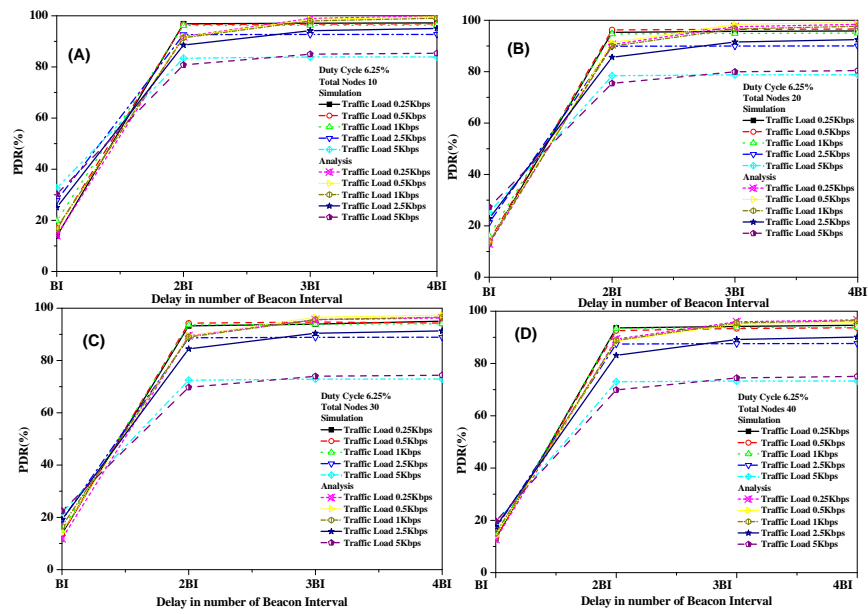

Fig. 3. PDR (\%) against burst number for both Simulation Results and Analytical Model at traffic load $0.25 \mathrm{Kbps}, 0.5 \mathrm{Kbps}, 1 \mathrm{Kbp}, 2.5 \mathrm{Kbps}$ and $5 \mathrm{Kbps}$ for Duty Cycle $6.25 \%$ as a function of total participating nodes (A) 10, (B) 20, (C) 30 and (D) 40 . 

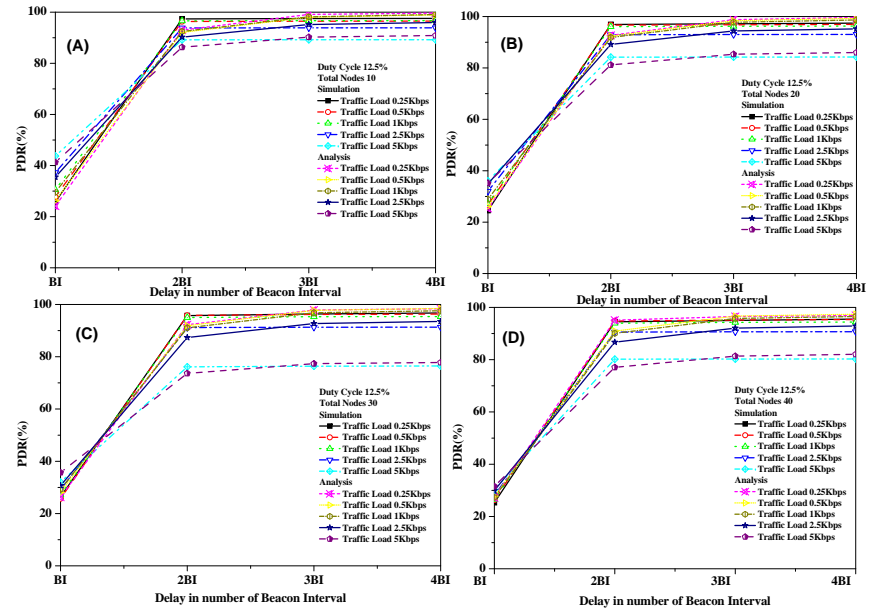

Fig. 4. PDR (\%) against burst number for both Simulation Results and Analytical Model at traffic load $0.25 \mathrm{Kbps}, 0.5 \mathrm{Kbps}, 1 \mathrm{Kbp}, 2.5 \mathrm{Kbps}$ and $5 \mathrm{Kbps}$ for Duty Cycle $12.5 \%$ as a function of total participating nodes (A) 10, (B) 20, (C) 30 and (D) 40.
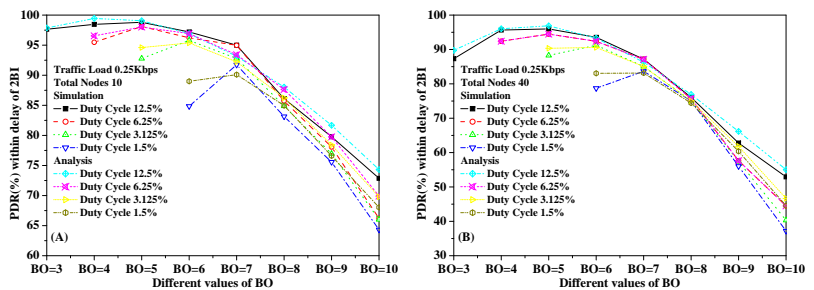

Fig. 5. PDR (\%) against $\mathrm{BO}$ values of 3, 4, 5, 6, 7, 8, 9 and 10 for both Simulation Results and Analytical Model at traffic load $0.25 \mathrm{Kbps}$ for Duty Cycle $12.5,6.25 \%, 3.125 \%$ and $1.5 \%$ as a function of total number of nodes (A) 10 and (B) 40 .
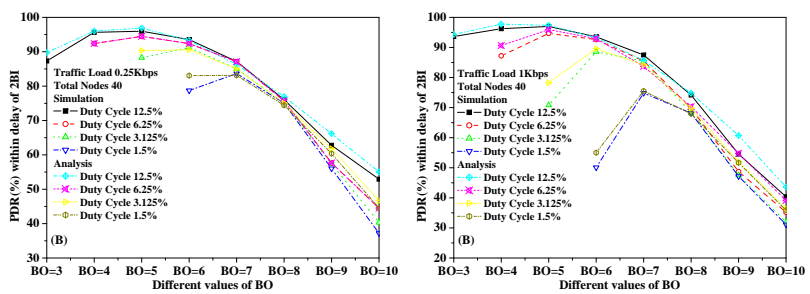

Fig. 6. PDR (\%) against BO values of 3, 4, 5, 6, 7, 8, 9 and 10 for both Simulation Results and Analytical Model at traffic load $1 \mathrm{Kbps}$ for Duty Cycle $12.5,6.25 \%, 3.125 \%$ and $1.5 \%$ as a function of total number of nodes (A) 10 and (B) 40 .

To validate the expression derived from the analytical model to compute PDR within a given latency we conduct a set of simulation experiments for different traffic loads at different duty cycles. Considering the low traffic applications and our aim of energy efficiency the ranges of both traffic load and duty cycle are kept low. The traffic load is kept within $2 \%$ of the channel capacity, i.e. 5 $\mathrm{Kbps}$, and the duty cycle is kept within $12.5 \%$. Total number of contending sensor nodes considered for the experiments are 10, 20, 30 and 40 .

In a slotted IEEE 802.15.4 network the packets arrive at the des-
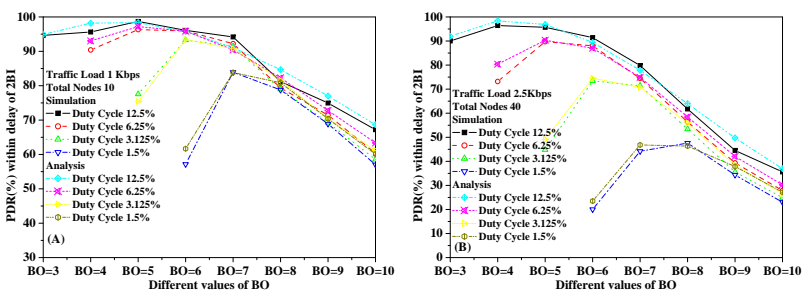

Fig. 7. PDR (\%) against BO values of 3, 4, 5, 6, 7, 8, 9 and 10 for both Simulation Results and Analytical Model at traffic load 2.5Kbps for Duty Cycle $12.5,6.25 \%, 3.125 \%$ and $1.5 \%$ as a function of total number of nodes (A) 10 and (B) 40

tinations during the active period of a cycle. For low duty cycle operations these deliveries occur in short bursts. The latency of the packets in the $k^{t h}$ burst will lie in the latency range of $(k-1) b i$ and $k b i$. Considering this we plot the PDR against latency expressed in terms of number of beacon intervals. The PDRs for both, the simulated results and the value computed from the analytical model, in Fig 2, Fig 3 and Fig 4 for $\mathrm{BO}=6$. It can be observed from the plots that the analytical model match quite well with the simulation results for different beacon orders as well as WSN sizes. It is interesting to observe in Fig 2 Fig 3 and Fig 4 that, both in case of analytical model as well as simulation results the growth of PDR against latency will be high initially and then taper down as the PDR approaches a saturation level. We call the latency point where saturation sets in as the Throughput Saturation Latency (TSL). The yield in PDR beyond TSL is marginal in comparison to the increase in latency. Therefore it will be prudent to consider the PDR at the $T S L$ as the PDR of the WSN.

Further, based on these results it can be observed that for all the cases, except one, the saturation sets in at $2 b i$ and therefore the TSL is $2 b i$. In the case of Fig 2 (A), (B), (C) and (D) for duty cycle of $3.125 \%$ and traffic load of 5 Kbps the above saturation does not occur. In this case the traffic load is very close to the available data rate of the channel and hence the data rate cannot be considered to be low enough for the duty cycle assumed and hence not appropriate. The above result can be explained as follows. During the first beacon interval the PDR remains low as a large fraction of the packets get generated within the inactive period and will be deferred to the following active period. Most of these deferred packets get successfully delivered within that active period when the data generation rate is low. The latency suffered by these successfully delivered packets is at most $2 b i$. As a result the increase in the fraction of packets with latency beyond $2 b i$ is therefore marginal. Hence the TSL for low traffic rate remains $2 b i$, except when the duty cycle value is such that the data rate is comparable to the bandwidth available or higher.

We repeat the experiment with beacon order values of $\mathrm{BO}=3,4,5$, $6,7,8,9$ and 10 with total numbers of sensor nodes taken as 10 , and 40 to verify the model for different beacon orders. Here the packets delivered within two BIs are only considered as most of the packets are delivered within two BIs. We plot both the results in Fig 5 , Fig 6 and Fig 7 for traffic loads of $0.25 \mathrm{Kbps}, 1 \mathrm{Kbps}$ and $2.5 \mathrm{Kbps}$ respectively.It can be observed from Fig 5 , Fig 6 and Fig 7 that the PDR values computed from the model and the simulation results match quite closely.

\subsection{Validation of expression for energy consumption:}

For the validation of the expression for energy consumption derived in the simulation model we compute the eEnergy per byte 

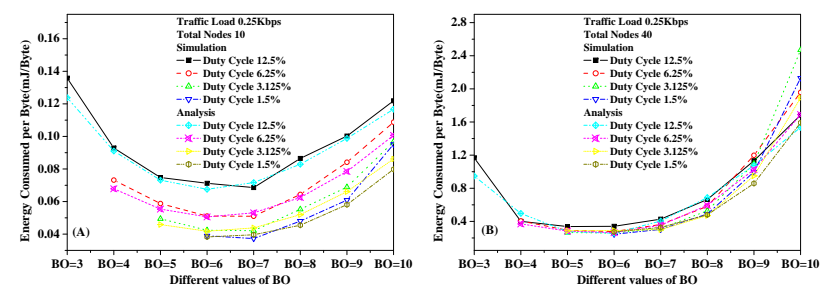

Fig. 8. $E P B D D$ against $\mathrm{BO}$ values of 3, 4, 5, 6, 7, 8, 9 and 10 for both Simulation Results and Analytical Model at traffic load $0.25 \mathrm{Kbps}$ for Duty Cycle $12.5,6.25 \%, 3.125 \%$ and $1.5 \%$ as a function of total number of nodes (A) 10 and (B) 40 .
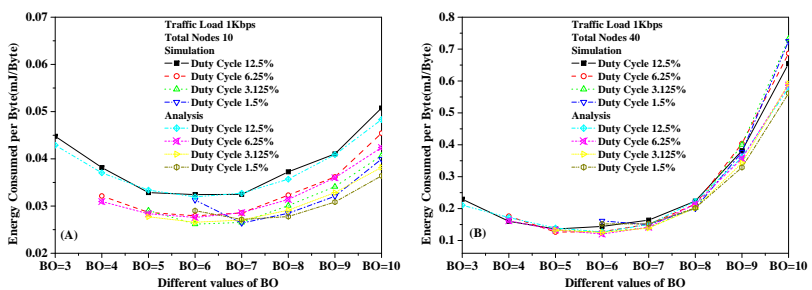

Fig. 9. $E P B D D$ against $\mathrm{BO}$ values of 3, 4, 5, 6, 7, 8, 9 and 10 for both Simulation Results and Analytical Model at traffic load $1 \mathrm{Kbps}$ for Duty Cycle $12.5,6.25 \%, 3.125 \%$ and $1.5 \%$ as a function of total number of nodes (A) 10 and (B) 40 .
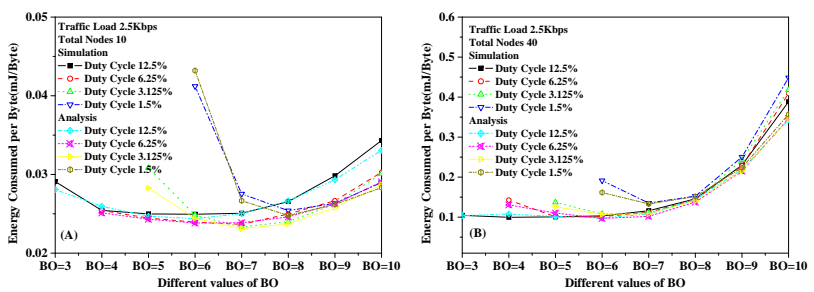

Fig. 10. $E P B D D$ against $\mathrm{BO}$ values of $3,4,5,6,7,8,9$ and 10 for both Simulation Results and Analytical Model at traffic load 2.5Kbps for Duty Cycle $12.5,6.25 \%, 3.125 \%$ and $1.5 \%$ as a function of total number of nodes (A) 10 and (B) 40 .

of data delivered (EPBDD) of the network for packets delivered within TSL through simulation as well as for the model for WSNs of sizes of 10 and 40 nodes and BO values of 3, 4, 5, 6, 7, 8 and 10 and plot these results in Fig 8 . Fig 9 and Fig 10 for traffic loads of $0.25 \mathrm{Kbps}, 1 \mathrm{Kbps}$ and $2.5 \mathrm{Kbps}$. It can be observed that analytical model results match quite well with the simulation results.

Further, it can also be observed from Fig 8 . Fig 9 and Fig 10 that the energy consumed initially decreases with increasing value of $\mathrm{BO}$, flattens a bit and then starts rising again. Higher energy consumption at very low $\mathrm{BO}$ values occurs due to the higher levels of retransmissions due to too short active period. At large $\mathrm{BO}$ values too energy inefficiency occurs due to retransmissions, but as a result of excessive deferred packets due to long inactive periods. At small values of $\mathrm{BO}$ another factor that degrade PDR and energy efficiency is that the coordinator needs to broadcast the beacon frame very frequently. This frequent transmission of beacon frame reduces the available bandwidth. The frequent beacon frames also force each node's radio to frequently wake up to receive these broadcast frames and acknowledge the coordinator causing additional energy consumption in the sensor nodes.

\section{CONCLUSION}

In this paper we have presented a Markov chain based analytical model for slotted IEEE 802.15.4 MAC protocol with duty cycle mechanism and derived expressions to compute PDR within a given latency limit, and energy consumed in a WSN per byte of data delivered. The model also allows computation of the energy consumed per byte of packet delivered (EPBDD) within the specified latency. The computation can be carried out for different duty cycles and different beacon orders at given traffic loads. We have validated the model through simulation experiments in NS2 environment. The results from simulation are found to agree well with the proposed model.

In this study we have also introduced the concept of Throughput Saturation Latency (TSL) that help us in achieving an optimal throughput within a given latency.

The proposed model shall be useful in determining the $P D R$ achievable within given latency and $E P B D D$ in a network at different settings of MAC parameters such as duty cycles, beacon orders etc. for different traffic loads. Thus the model can be used to choose the right MAC parameter values for a given application with a given traffic load such that the required PDR is achieved within latency limit of the application while the EPBDD is minimized.

\section{REFERENCES}

[1] Giuseppe Bianchi. Performance analysis of the IEEE 802.11 distributed coordination function. Selected Areas in Cоттиnications, IEEE Journal on, 18(3):535-547, 2000.

[2] Bruno Bougard, Francky Catthoor, Denis C Daly, Anantha Chandrakasan, and Wim Dehaene. Energy efficiency of the IEEE 802.15. 4 standard in dense wireless microsensor networks: Modeling and improvement perspectives. In Design, Automation, and Test in Europe, pages 221-234. Springer, 2008.

[3] Bo Gao, Chen He, and Lingge Jiang. Modeling and analysis of ieee 802.15. $4 \mathrm{csma} / \mathrm{ca}$ with sleep mode enabled. In Communication Systems, 2008. ICCS 2008. 11th IEEE Singapore International Conference on, pages 6-11. IEEE, 2008.

[4] Yu-Kai Huang, Ai-Chun Pang, and Hui-Nien Hung. A comprehensive analysis of low-power operation for beaconenabled IEEE 802.15. 4 wireless networks. Wireless Communications, IEEE Transactions on, 8(11):5601-5611, 2009.

[5] IEEE TG 15.4, part 15.4: Wireless Medium Access Control (MAC) and Physical Layer (PHY) Specifications for LowRate Wireless Personal Area Networks (WPANs), IEEE Std., New York.

[6] Gang Lu, Bhaskar Krishnamachari, and Cauligi S Raghavendra. Performance evaluation of the IEEE 802.15. 4 MAC for low-rate low-power wireless networks. In Performance, Computing, and Communications, 2004 IEEE International Conference on, pages 701-706. IEEE, 2004.

[7] Jelena Mišić, Shairmina Shafi, and Vojislav B Mišić. The impact of MAC parameters on the performance of 802.15. 4 PAN. Ad Hoc Networks, 3(5):509-528, 2005.

[8] Network Simulator version - 2. http://www.isi.edu/ nsnam/ns/

[9] Pan Gun Park, Piergiuseppe Di Marco, Carlo Fischione, and Karl Henrik Johansson. Delay distribution analysis of wireless personal area networks. In CDC, pages 5864-5869, 2012.

[10] Pangun Park, Piergiuseppe Di Marco, Pablo Soldati, Carlo Fischione, and Karl Henrik Johansson. A generalized markov 
chain model for effective analysis of slotted ieee 802.15. 4. In Mobile Adhoc and Sensor Systems, 2009. MASS'09. IEEE 6th International Conference on, pages 130-139. IEEE, 2009.

[11] The ZigBee Specification version 2.0. http://www . zigbee.org/Standards/Downloads .aspx

[12] Andreas Willig. Recent and emerging topics in wireless industrial communications: A selection. Industrial Informatics, IEEE Transactions on, 4(2):102-124, 2008.

[13] Zhuoling Xiao, Chen He, and Lingge Jiang. Slot-based model for IEEE 802.15. 4 MAC with sleep mechanism. Communications Letters, IEEE, 14(2):154-156, 2010.

[14] Richard Zurawski. The industrial communication technology handbook. CRC Press, 2005. 